\title{
Hacia una economía de plataformas responsable
}

\author{
Towards a responsible platform economy
}

\author{
Albert Cañigueral Bagó \\ Connector Ouishare para España y América Latina
}

\begin{abstract}
Resumen
La economía de plataformas ha emergido con mucha fuerza en una amplia diversidad de sectores productivos (movilidad, turismo, finanzas, etc.). Estas plataformas están demostrando ser más eficientes desde un punto de vista de gestión, ofrecen más oportunidades de intercambio de valor entre los ciudadanos y reducen la huella ecológica en algunos casos. El análisis completo debe incorporar las externalidades negativas de las plataformas, así como los retos regulatorios (licencias, laboral, tributos, etc.). Presentamos diversas herramientas y reflexiones que nos facilitan este análisis más completo al parametrizar una plataforma digital para medir su nivel de responsabilidad social.
\end{abstract}

Palabras clave: economía colaborativa, economía compartida, plataformas digitales, economía de plataformas, cooperativismo de plataforma, regulación.

Clasificación JEL: D13, L81, K2, O35.

\begin{abstract}
The platform economy has emerged with great force in a wide diversity of productive sectors (mobility, tourism, finance, etc.). These platforms are proving to be more efficient from a management point of view, offer more opportunities to exchange value among citizens and reduce the ecological footprint in some cases. The complete analysis should incorporate the negative externalities of the platforms and the challenges related to their regulation (licenses, labor, taxes, etc.). We present several tools and reflections that facilitate this more complete analysis when parameterizing a digital platform to measure its level of social responsibility.
\end{abstract}

Keywords: collaborative economy, sharing economy, digital platforms, platform economy, platform cooperativism, regulation.

JEL classification: D13, L81, K2, O35.

\section{Introducción}

En un periodo breve de tiempo la economía de plataformas ha impactado de manera relevante en la vida de millones de personas alrededor del mundo. Diversos estudios y encuestas indican que, entre la población europea, existe un grado de uso superior al 30 por 100 y un grado de conocimiento superior al 50 por 100, siendo la segunda mano, los viajes compartidos en coche y los alojamiento turísticos los más 
populares (CNMC Panel de Hogares $2017^{1}$, Flash Eurobarometer 467², Single Market Scoreboard Collaborative Economy ${ }^{3}$ ).

Ya en 2010, la revista Time Magazine incluyó el llamado «consumo colaborativo» como una de las diez ideas que iban a cambiar el mundo ${ }^{4}$ y el libro What's Mine is Yours ${ }^{5}$ repasó las bondades de las plataformas desde el punto de visto económico, social y medioambiental.

Con el paso de los años hemos podido experimentar varios de los accidentes integrales ${ }^{6}$ derivados de las plataformas digitales y su crecimiento exponencial: externalidades negativas, dificultad para aplicar las normativas existentes o el pago de los correspondientes tributos para mencionar aquello más mediático. Publicaciones como Lo tuyo es mío: contra la economía colaborativa ${ }^{7}$ resumen esa crítica hacia este complejo fenómeno.

Este documento presenta, en la segunda sección, los principales conceptos y definiciones de la economía de plataformas y su desarrollo actual en varios sectores. La tercera sección ofrece un balance entre los beneficios y perjuicios del rápido desarrollo de estas plataformas. Por último, la cuarta sección explora la diversidad de actores de la economía de plataformas, más allá de las startups más conocidas, y presenta diversos marcos de referencia para evaluar el grado de responsabilidad de las plataformas digitales respecto al conjunto de sus impactos. El trabajo se cierra con una última sección en la que se ofrecen unas breves conclusiones.

\section{La economía de plataformas: el futuro nunca estuvo tan presente}

\subsection{Definiciones}

Consumo colaborativo, sharing economy, economía colaborativa, economía $p 2 p$, gig economy, economía del acceso, etc.: uno de los primeros retos a los que nos enfrentamos al hablar de este fenómeno es la propia nomenclatura que ha generado, dado que es compleja, diversa e incluso contradictoria. Traduciendo el título de un artículo de Rachel Botsman: «La economía colaborativa no tiene una definición compartida» ${ }^{8}$.

\footnotetext{
${ }^{1}$ Ver https://blog.cnmc.es/2017/11/02/panel-de-hogares-cnmc-enganchados-las-apps-de-compraventade-segunda-mano/.

2 Ver https://data.europa.eu/euodp/data/dataset/S2184_467_ENG.

${ }^{3}$ Ver http://ec.europa.eu/internal_market/scoreboard/performance_per_policy_area/collaborative-economy/index_en.htm.

${ }^{4}$ Ver Today's Smart Choice: Don't Own. Share. http://content.time.com/time/specials/packages/article/0,28804,2059521_2059717_2059710,00.html.

5 Ver What's Mine Is Yours: The Rise of Collaborative Consumption. https://www.harper-collins. com/9780061963544/whats-mine-is-yours/.

${ }^{6}$ Ver The integral accident. https://en.wikipedia.org/wiki/Paul_Virilio\#The_integral_accident.

${ }^{7}$ Ver What's Yours is Mine: Against the Sharing Economy. http://tomslee.net/category/my-books/whatsyours-is-mine.

${ }^{8}$ Ver https://www.fastcompany.com/3022028/the-sharing-economy-lacks-a-shared-definition.
} 
Aunque existe mucho debate y literatura al respecto, para este documento tomamos como referencia las definiciones propuestas por el informe Los modelos colaborativos y bajo demanda en plataformas digitales (Rodríguez, 2017) de la Asociación Española de la Economía Digital, a saber:

- Economía colaborativa. La economía colaborativa la conforman aquellos modelos de producción, consumo o financiación que se basan en la intermediación entre la oferta y la demanda generada en relaciones entre iguales ( peer to peer, $\mathrm{P} 2 \mathrm{P}$ o business to business, $\mathrm{B} 2 \mathrm{~B}$ ) o de particular a profesional a través de plataformas digitales que no prestan el servicio subyacente, generando un aprovechamiento eficiente y sostenible de los bienes y recursos ya existentes infrautilizados $^{9}$, permitiendo utilizar, compartir, intercambiar o invertir los recursos o bienes, pudiendo existir o no una contraprestación económica entre los usuarios.

Esta definición puede generar algo de controversia, ya que el uso del adjetivo «colaborativo» genera una expectativa de que estas plataformas tengan una orientación poco o nada lucrativa (modelos de intercambio, regalo, compartición de gastos, etc.), un modelo de gobernanza participativa o que usen datos y tecnología abierta. Lo cierto es que en muchos casos las plataformas de la economía colaborativa no cumplen esas expectativas y por ello se generan debates acerca de lo que es o no es «economía colaborativa».

- Economía bajo demanda o gig-economy. Dentro de la economía bajo demanda (a menudo también referida como gig-economy), encontramos aquellos modelos de consumo y provisión de servicios que se basan en la intermediación entre la oferta y la demanda generada habitualmente de profesional a consumidor (business to consumer, B2C) a través de plataformas digitales que no prestan el servicio subyacente. La prestación se origina en base a las necesidades del usuario que demanda y se adapta a sus preferencias, presentándose normalmente a cambio de una contraprestación y habitualmente con ánimo de lucro.

La diferencia fundamental de este tipo de modelos bajo demanda y los modelos colaborativos es que entre los usuarios existe una relación comercial, es decir, son plataformas en las que tiene lugar la prestación de un servicio ya sea por parte de profesionales o por parte de particulares, dependiendo del modelo.

- Economía de acceso. Consideramos que forman parte de la economía de acceso aquellos modelos de consumo en los cuales una empresa, con fines comerciales, pone a disposición de un conjunto de usuarios unos bienes para su uso temporal, adaptándose al tiempo de uso efectivo que requieren dichos usuarios y flexibilizando la localización espacial de los mismos.

\footnotetext{
${ }^{9}$ La interpretación de recurso infrautilizado debe hacerse de manera amplia, entendido como recurso no solamente un bien, como un vehículo o un inmueble, sino también el tiempo, el conocimiento o incluso el dinero.
} 
Si bien la economía colaborativa ya fomenta el acceso frente a la propiedad de los bienes, la economía de acceso hace referencia únicamente a los modelos en los que la plataforma digital presta el servicio subyacente de manera centralizada y los usuarios normalmente no tienen contacto directo entre sí para efectuar las transacciones.

Para abarcar la complejidad y la dimensión del fenómeno en su conjunto este documento prefiere usar el término neutral de «economía de plataformas» ${ }^{10}$. «Economía de plataformas» es también la terminología que organismos como la Unión Europea $^{11}$, la OCDE ${ }^{12}$ o el World Economic Forum ${ }^{13}$ han adoptado.

\subsection{Usos de la economía de plataformas en diversidad de sectores}

El uso de las plataformas digitales ya impacta de manera transversal en muchos ámbitos de la sociedad. En el fondo se trata de digitalizar las relaciones sociales y comerciales que se dan en el marco de las actividades económicas y sociales.

Las plataformas están alterando la naturaleza de la competencia, el orden jerárquico de los jugadores en muchos sectores, creando industrias y categorías completamente nuevas, haciendo colapsar los mercados existentes y abriendo otros nuevos. El impacto de este modelo organizativo hizo que Robin Chase, fundadora de Zipcar, afirmara: «Cualquier negocio que pueda convertirse en plataforma se convertirá en plataforma» ${ }^{14}$.

\subsubsection{Alojamiento y turismo}

El alojamiento y el turismo facilitado mediante plataformas han sido sectores punteros de la economía de plataformas alrededor del mundo. La modalidad más conocida es el de alquiler de habitaciones o pisos enteros de particulares para estancias de corta duración. La plataforma americana Airbnb es la referencia en este ámbito con competidores de menor tamaño como HomeAway, FlipKey, VRBO, Wimdu o Aluguest. Existen también plataformas especializadas en verticales concretos como MisterBnB, para el público homosexual. Se están popularizando metabuscadores como Tripping o Apartum que ofrecen resultados agregados de las búsquedas en Airbnb y

10 El libro Platform Revolution, del profesor Sangeet Paul Choudary; el estudio «Pentagrowth: las cinco palancas del crecimiento exponencial», de la consultora Ideas For Change, y la metodología «Platform Design Toolkit», de Simone Cicero, son excelentes textos para profundizar en la compresión de las plataformas digitales.

${ }^{11}$ Ver Government Responses to the Platform Economy: Where Do We Stand? https://papers.ssrn.com/ sol3/papers.cfm?abstract_id=3055062.

12 Ver Rethinking Antitrust Tools for Multi-Sided Platforms 2018. http://www.oecd.org/competition/ rethinking-antitrust-tools-for-multi-sided-platforms.htm.

13 The 'Platform Economy' has arrived. Here's what you need to know about it. https://www.weforum. org/agenda/2016/07/platform-economy-boon-or-doom/.

${ }_{14}$ Ver https://wtfeconomy.com/everything-that-can-become-a-platform-will-become-a-platform-216bcf b89855?gi=d3eb23f197a9 
el resto de páginas. También otras variantes en relación al alojamiento son el intercambio de casas (GuestToGuest) o el alojamiento gratuito (Couchsurfing).

Además de alojarse, los turistas quieren conocer el territorio que visitan y es por ello que algunas de las plataformas están incorporando experiencias turísticas locales (tours, deportes, gastronomía, conciertos, cultura, etc.) acompañados o liderados por particulares. Airbnb Experiences, SofaConcerts o GuruWalk son ejemplos de este segmento.

Aunque los conflictos habituales en el ámbito del alojamiento son con el status quo del sector hotelero local, desde un punto de vista de las ciudades, es importante ir más allá en el análisis y estudiar el impacto de estas plataformas en lo relativo al acceso a la vivienda ${ }^{15}$ (alquiler y compra) por parte de los ciudadanos, sobre todo cuando la oferta de alojamiento turístico se profesionaliza ${ }^{16}$. En el lado positivo hay que ver cómo estas plataformas distribuyen de manera más horizontal los beneficios económicos de la actividad turística entre un mayor número de personas ${ }^{17}$.

\subsubsection{Movilidad y transporte}

La primera experiencia en economía de plataformas es habitualmente mediante una plataforma relacionada con la movilidad. Una bicicleta pública compartida (Bicing en Barcelona o EcoBici México), un vehículo de carsharing (Zipcar, Car2go, ECooltra, etc.), un servicio tipo Uber o Cabify, o un trayecto en coche compartido entre ciudades (BlaBlacar o Carpoolear). Estos servicios nos permiten ser conscientes de que para desplazarnos no necesitamos hacerlo siempre en un vehículo de nuestra propiedad.

Otra posibilidad es la de alquilar vehículos de otras personas que no los usan (coches, furgonetas, campervans, etc.) en plataformas como SocialCar, Drivy o Carengo e incluso en modalidad cooperativa como SomMobilitat. Para minimizar riesgos, diversas empresas de seguros ya han desarrollado productos a medida para este tipo de servicios.

Ante la gran cantidad de opciones disponibles de movilidad mediante plataformas digitales, la tendencia futura es la de simplificar la experiencia de usuario mediante la «movilidad como servicio» ${ }^{18}$. Se agrupan la mayoría de las opciones de transporte en el territorio en una única aplicación como RAAC Trips o CityMapper. Idealmente el pago de los diversos servicios se puede realizar con un sistema integrado de tarifas.

15 Ver controversias «Los informes que refutan a la CNMC: Airbnb sí infla el precio de la vivienda en EE UU».https://elpais.com/economia/2018/08/14/actualidad/1534253546_081610.html.

16 Ver «El sueño de Airbnb cae en manos de los profesionales». https:/elpais.com/economia/2018/ 01/19/actualidad/1516386039_221435.html.

17 Ver https://www.airbnb.com/economic-impact.

$18 \mathrm{Ver}$ https://blogs.iadb.org/moviliblog/2017/11/02/movilidad-como-servicio-maas-como-hacer-paraconectar-el-caso-de-finlandia/. 
Las ciudades deben comprender el impacto, las oportunidades y los retos de estas modalidades de transporte en ámbitos como:

- El trasvase de usuarios desde el transporte público y/o desde el vehículo privado a estos medios de transporte.

- La congestión de las vías y la polución, tanto al añadir vehículos para Uber o Cabify pero también al compartir trayectos en estos mismos vehículos mejorando la ocupación media.

- El uso del espacio público en casos como ECooltra (motocicletas eléctricas) o Donkey Republic (bicicletas compartidas) al estacionar los vehículos.

- El uso de los datos de estas plataformas para un mejor conocimiento y planificación de la movilidad urbana e interurbana.

Los principales conflictos en el ámbito de la movilidad y las plataformas digitales se observan en las licencias de taxi respecto a las licencias de VTC. En España en 2018 la gestión de estas licencias pasó a ser competencia de las comunidades autónomas y los ayuntamientos, generando fragmentación regulatoria. Esta diversidad de regulaciones ha sido el origen de los conflictos que han llevado a la huelga al sector del taxi tradicional y a que empresas como Uber y Cabify hayan cesado sus operaciones en algunas ciudades de España.

Las empresas fabricantes de vehículos (Renault, Daimler, Seat, etc.) han reaccionado a estos nuevos escenarios, bien desarrollando sus propias plataformas (Car2Go de Daimler, Emov de Peugeot-Citroën, Zity de Nissan) o bien comprando e integrando algunas de las startups de mayor éxito ${ }^{19}$. La tendencia es que las empresas están pasando de vender productos (vehículos) a vender servicios (movilidad integrada).

\subsubsection{Finanzas}

Las plataformas digitales de financiación colectiva ${ }^{20}$ (crowdfunding) facilitan la obtención del capital necesario para proyectos personales, de empresas e incluso del sector público. La aportación económica realizada al proyecto puede ser a cambio de recompensas relacionadas con el proyecto (Verkami), de unos derechos de participación sobre el proyecto (Crowdcube), de un interés sobre el préstamo del dinero (Arboribus) o como un donativo altruista (GoFundMe).

Para que el encuentro entre las personas que proponen proyectos y las personas que quieran financiarlos sea más eficaz surgen plataformas especializadas en ámbitos concretos. Destacar el crowdfunding de proximidad (Tudigo para negocios loca-

19 Ver https://www.cbinsights.com/research/auto-corporates-investment-ma-timeline/.

${ }^{20}$ Ver informe sobre Crowdfunding en España 2017 (Crowdfunding, 2018), aporta datos actualizados del sector. https://www.universocrowdfunding.com/el-crowdfunding-recaudo-en-espana-101-millones-deeuros-en-2017/ 
les) y el crowdfunding cívico ${ }^{21}$ (SpaceHive o La Ruche). También existen plataformas de crowdfunding orientadas a proyectos sociales (Ecrowd) y a la creación de procomún ${ }^{22}$ ciudadano (Goteo).

Más allá de la financiación colectiva, otro ámbito de interés para el desarrollo de la economía de plataformas son las monedas locales con el objetivo de promover y reforzar la economía local. Tradicionalmente las monedas locales han surgido en papel. Ahora, con aplicaciones como la israelí Colu o la argentina MonedaPar, se están digitalizando, lo que facilita su adopción por parte de comercios y habitantes del territorio.

Uno de los potenciales más destacados de las finanzas basadas en plataformas digitales es que pueden ser una herramienta de inclusión financiera, ya sea mediante mecanismos de crowdfunding ${ }^{23} \mathrm{o}$ bien mediante el uso de blockchain $^{24}$. El proyecto Systema $\mathrm{D}^{25}$ liderado por la ONG Bitcoin Argentina ${ }^{26}$ es un buen ejemplo de ello.

\subsubsection{Compraventa de segunda mano}

Aplicaciones de compraventa de productos de segunda mano como Wallapop o Vibbo en España, o bien LetGo o OLX en varios países de América Latina, son usadas a diario por millones de personas. Además de las mejoras tecnológicas es relevante que, desde un punto de vista cultural, el estigma de los productos de segunda mano en el pasado ha dado paso a su normalización actual. El Panel de Hogares de la CNMC 2017 (CNMC , 2017) destaca que las plataformas digitales de compraventa en España son usadas por casi un 30 por 100 de la población, como mínimo una vez al año.

Complementando a las plataformas de productos de segunda mano de carácter generalista se han desarrollado también servicios especializados como Chicfy (solo ropa), Manzanas Usadas (productos de Apple segunda mano), StubHub (entradas para eventos), YoNoDesperdicio (desperdicio alimentario), etc.

\subsubsection{Tareas y trabajo}

Las plataformas digitales están transformando rápida y profundamente los mercados laborales. Las plataformas permiten contratar a personas por periodos cortos de duración y para tareas muy concretas, sin que llegue a mediar un contrato de trabajo tradicional. Es lo que hemos definido al inicio de esta sección como gig economy o economía bajo demanda.

\footnotetext{
${ }^{21}$ Ver http://wiki.p2pfoundation.net/Civic_Crowdfunding.

22 Bien comunal.

23 Ver «Papel del crowdfunding en la nueva política de inclusión financiera». https://aficomx.wordpress. com/2016/07/17/papel-del-crowdfunding-en-la-nueva-politica-de-inclusion-financiera-de-mexico/.

24 Sistema de seguridad formado por bloques alojados en una base de datos compartida.

25 Ver http://systemad.org/.

26 Ver https://www.bitcoinargentina.org.
} 
Como se observa en la figura sobre la plataformización de las relaciones laborales (Figura 1), existen plataformas para una amplia diversidad de tareas. Desde Amazon Mechanical Turk (micro tareas online), a Glovo, Deliveroo o Uber Eats (para entregas físicas de trabajos de corta duración), JobToday o CornerJob (muy orientado al sector HORECA, venta minorista y eventos), Freelancer o UpWork (diseño, programación, traducción), hasta plataformas para talento de élite (analistas, financieros, CEO) como TopTal.

La cantidad de personas que usan estas plataformas hoy en día aún es limitada (Pesole, Urzi-Brancati, Fernández-Macías, Biagi y González-Vázquez, 2018), pero en un futuro cercano las oportunidades de generación de ingresos para una creciente mayoría de ciudadanos van a estar mediadas por este tipo plataformas digitales laborales. Observando esta tendencia es recomendable:

- Acompañar y capacitar en el uso de estas plataformas a estos nuevos «trabajadores independientes», por ejemplo con programas específicos dentro de los programas para emprendedores y/o personas desempleadas.

- Observar las oportunidades de realizar trabajos completamente online (UpWork) o venta de productos locales en remoto (Esty) para los territorios periurbanos y rurales.

- Fomentar que las empresas tradicionales, centros técnicos y centros de formación adapten sus planes de formación para este nuevo perfil laboral y este nuevo estilo de contratación y gestión de talento.

FIGURA 1

\section{LA PLATAFORMIZACIÓN DE LAS RELACIONES LABORALES}

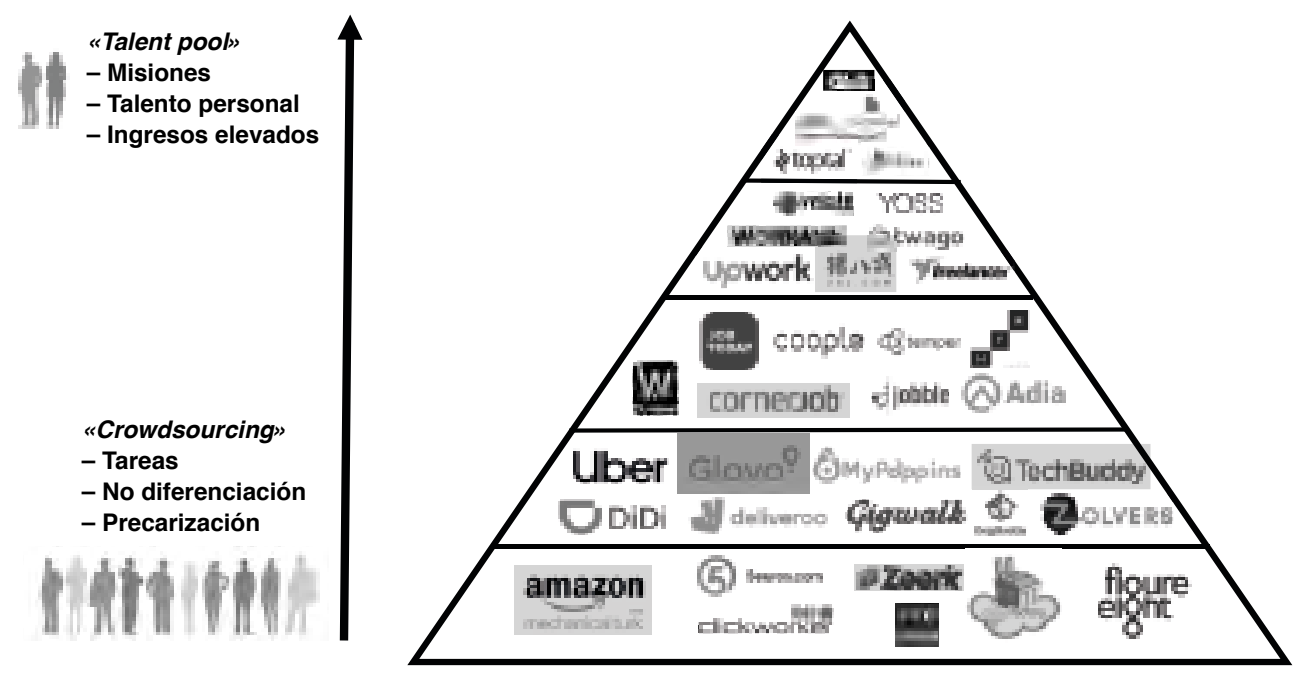

FUENTE: Elaboración propia. 
En este ámbito del trabajo, destacar finalmente el fuerte crecimiento del movimiento coworking (espacios de trabajo compartido) alrededor del mundo y la aparición de los nómadas digitales ${ }^{27}$ (personas de trabajan de manera remota desde cualquier ubicación). A caballo entre el ámbito del trabajo y el del alojamiento están los llamados colivings o coviviendas, una clase de comunidad intencional compuesta de casas privadas complementadas con extensos espacios comunitarios.

\subsubsection{Conocimiento y educación}

En el mundo digital el conocimiento ya no es algo exclusivo y de difícil acceso. Ahora el conocimiento está online, disponible a menudo de manera gratuita, y cualquiera puede ser profesor o alumno en temáticas muy específicas.

Las plataformas para educación y conocimiento online reciben el nombre de MOOC (Massive Open Online Courses): cursos masivos abiertos online basados en un formato de «curso» con profesores «dando clase» (en video). Plataformas como Coursera, EdX o Udacity son las de referencia. En castellano destacan MiriadaX o el portal de la UNED Abierta.

Como complemento a estos cursos de duración media o larga se han popularizado también las cápsulas formativas de corta duración en ámbitos más específicos, disponibles en servicios como por ejemplo Tutellus. Se compra una píldora individual o bien se realiza una suscripción mensual para acceder a todo el catálogo de contenidos de manera ilimitada.

El proyecto Khan Academy ha sido muy galardonado. Khan Academy ofrece más de 5.000 video-lecciones de menos de diez minutos en YouTube acompañadas de ejercicios. Los contenidos están sobre todo enfocados a escolares de enseñanza primaria y secundaria en ámbitos como matemáticas, biología, química, física, e incluso de humanidades como finanzas o historia.

En algunos casos las plataformas se orientan a un nicho concreto de formación, como el caso de Duolingo o Busuu para el aprendizaje de idiomas. En estos casos, más allá de los contenidos, también se fomenta la interacción entre los participantes en foros y video llamadas.

Para certificar la obtención de estos conocimientos, proyectos como Open Badges (que está siendo usado por el servicio médico inglés $\mathrm{NHS}^{28}$ ) o más recientemente Smart Degrees, basado en blockchain y con la Universidad Carlos III de Madrid como socio académico, surgen como alternativas a la certificación de los centros formativos tradicionales.

Finalmente, mencionar la Wikipedia como el proyecto más importante de plataformas digitales procomún. En este proyecto el conocimiento es co-creado y

\footnotetext{
27 Ver https://www.dnxhub.com/.

${ }^{28}$ Ver https://www.openbadgeacademy.com/NHS.
} 
co-validado por los usuarios y se ofrece bajo licencias abiertas Creative Commons ${ }^{29}$. Las herramientas Wiki que se usan son de código abierto y la gobernanza de todo el proyecto es codiseñada con los propios usuarios bajo la supervisión de la Fundación Wikipedia.

\subsubsection{Alimentación y gastronomía}

La alimentación y la gastronomía son uno de los ámbitos de mayor potencial para la economía de plataformas en ciudades de tamaño intermedio, según el estudio Sharitories ${ }^{30}$. Las plataformas digitales han permeado ya en todos los puntos de la cadena de valor del sector de la alimentación y la gastronomía ${ }^{31}$.

En lo referente a la producción, existen bancos colectivos de semillas (Xarxa Catalana de Graners en Cataluña), se comparten conocimientos y técnicas agrícolas (DigitalGreen en India), se alquilan herramientas y vehículos para el trabajo en el campo (VotreMachine en Francia), etc.

En lo referente a la distribución y consumo existen proyectos como La Colmena Que Dice Sí (Francia) o Farmingo (EE. UU.), que simplifican la creación de grupos de consumo de proximidad y las compras colectivas mediante el uso de plataformas. También hay un creciente movimiento de supermercados cooperativos ${ }^{32}$ como La Louve (París) o la pionera Park Slope (NYC). Para la trazabilidad en la cadena de distribución alimentaria destacamos plataformas de código abierto como Open Food Networks o bien iniciativas basadas en blockchain como Provenance.

En la etapa de elaboración ya se comparten cocinas industriales y obradores en coworkings especializados en cocina, los cookworkings ${ }^{33}$. Pensando en aquellas personas a las que no les gusta cocinar, otras personas pueden cocinar en mayor cantidad y vender raciones a precio de coste (Share YourMeal) o bien ofrecer esas raciones en un sistema de intercambios (CoCooking).

Finalmente, en la etapa de consumo (sector restauración), plataformas como EatWith permiten organizar experiencias gastronómicas en casas particulares, es como un Airbnb para ir a cenar en casas de desconocidos. En esta actividad emergen conflictos respecto a las normativas sanitarias y las licencias de actividad. «Si están dados de alta, tienen el carné de manipulador de alimentos, pagan sus impuestos y tienen un registro sanitario, no hay problema, pero, de entrada, un particular no puede vender comidas», asegura Emilio Gallego, secretario general de la Federación Española de Hostelería (FEHR), en referencia a que la mayor parte de los anfitriones

\footnotetext{
${ }^{29}$ Ver https://creativecommons.org/.

${ }^{30}$ Ver https://www.sharitories.eu/publications/.

${ }^{31}$ El proyecto de investigación ShareCity (http://sharecity.ie/) recopila una amplia variedad de prácticas en este sector. El capítulo dedicado a la alimentación del proyecto Local Goverments and Sharing Economy (http://www.localgovsharingecon.com/) es muy completo.

${ }_{32}$ Ver http://www.eleconomista.es/economia/noticias/8896786/01/18/Supermercados-cooperativos-hacer-la-compra-en-el-negocio-de-todos.html.

${ }^{33}$ Ver http://www.theshedcoworking.com/cookworking/.
} 
registrados en estas plataformas son particulares que no cumplen con estos requisitos.

Aunque ya se mencionaron en el apartado sobre tareas y trabajo, las plataformas de delivery (GlovoApp, Uber Eats, Rappi, etc.) están también transformando la industria de la alimentación y restauración ${ }^{34}$. Algunos restaurantes están creando cocinas solo dedicadas al delivery: restaurantes $2.0^{35} \mathrm{o}$ dark kitchens sin camareros, mesas ni sillas.

Como última etapa de la alimentación, la comida sobrante entra en circuitos de redistribución mediante aplicaciones como Yo No Desperdicio (España) u Olio (Reino Unido).

\subsubsection{Salud}

«El envejecimiento de la población, el aumento de las enfermedades crónicas, un gasto sanitario que crece más rápido que el PIB, etc. Comienza a ser imprescindible para los diferentes gobiernos y administraciones repensar la asignación de los recursos sanitarios para garantizar la sostenibilidad de los sistemas sanitarios. En este contexto se observa a la tecnología digital y en pensamiento colaborativo como herramientas que pueden ayudar a resolver los problemas de coste-efectividad en el ámbito de la salud», Andrea Barbiero ${ }^{36}$ en la revista New Medical Economics de abril $2018^{37}$.

Los centros sanitarios optimizan el uso del equipamiento disponible mediante plataformas como Cohealo o Floow2Healthcare. Estas plataformas ahorran dinero a los sistemas de salud al compartir equipos entre instalaciones, aumentar la disponibilidad de equipos, eliminar alquileres y reducir los gastos de capital.

A medio camino entre el sector salud y el alojamiento para los familiares de los pacientes que necesitan quedarse cerca de los hospitales, Airbnb ha llegado a acuerdos en Milán ${ }^{38}$ para ofrecer alojamientos con fuertes descuentos. En Reino Unido, el servicio NHS está experimentado con un Airbnb para los pacientes ${ }^{39}$ que se recuperan de intervenciones quirúrgicas menores con tal de liberar camas del hospital para otros pacientes más graves.

\footnotetext{
${ }^{34}$ Ver https://www.elperiodico.com/es/economia/20180821/comida-domicilio-boom-6996997.

${ }^{35}$ Ver https://www.playgroundmag.net/food/sin-camareros-sin-mesas-sin-sillas-el-boom-de-los-restaurantes-fantasma_30016293.html.

${ }_{36}$ Andrea Barbiero es la fundadora del blog http://www.co-salud.com/.

${ }^{37}$ Ver http://www.newmedicaleconomics.es/index.php/articulos/articulos-de-interes/965-aplicando-logi ca-colaborativa-en-el-entorno-de-la-salud.

${ }^{38}$ Ver https://www.airbnbcitizen.com/airbnb-partners-with-milan-and-cadmi-to-provide-free-accomodations-for-medical-patients/.

${ }^{39}$ Ver http://www.co-salud.com/archivos/la-sanidad-britanica-pilota-airbnb-alquilar-habitaciones-pacientes/.
} 
Los doctores también están disponibles «bajo demanda» en aplicaciones como TelADoc o DoctorOnDemand. Un caso de uso muy interesante de estas aplicaciones es el de la telemedicina ${ }^{40}$, especialmente útil en zonas rurales y remotas.

Por el lado de los pacientes, las plataformas digitales también son útiles. Aquellos pacientes que comparten dolencias se agrupan y comparten igualmente información en plataformas como Patients Like Me o HelpAround. Los últimos avances en el ámbito de los pacientes se centran en plataformas de uso y gestión de los datos sanitarios de los pacientes en un formato de cooperativa y usando blockchain y smarts contracts para gestionar los permisos de uso de esos datos. Un proyecto de referencia es SalusCoop liderado por Ideas For Change desde Barcelona.

Como el ámbito de la salud es tan amplio, la diversidad de plataformas también lo es. Por un lado las personas cuidadoras para el hogar están disponibles en plataformas bajo demanda como Cuideo, Care.com o Room2Care. El reto es la certificación de estos profesionales, más allá de los sistemas de reputación que puedan incorporar las propias aplicaciones. Por otro lado, Hearts Milk Bank es una exitosa plataforma peer-to-peer para facilitar la donación de leche materna a bebés prematuros y enfermos en hospitales del Reino Unido.

\subsection{Algunas notas sobre la dimensión económica y de uso de la economía de plataformas}

La falta de una definición acordada y la dificultad para acceder a los datos de las plataformas hace muy complejo conocer en detalle el alcance real del fenómeno de la economía de plataformas. Podemos apuntar algunos datos o encuestas recientes que intentan aportar información al respecto:

- Los ingresos brutos en la Unión Europea de las plataformas colaborativas y sus proveedores se estimaron en 28.000 millones de euros en 2015. Se espera un impacto de 300.000 millones de euros en $2025^{41}$. En ese mismo año, se estima que muchas áreas de la economía colaborativa rivalizarán en tamaño con sus contrapartes tradicionales ${ }^{42}$.

- En 2016, el Eurobarómetro $438^{43}$ mostró que más de la mitad de la población de la Unión Europea tiene conocimiento acerca de la economía colaborativa. Una de cada seis personas ya es usuario de alguno de los servicios. Casi un tercio de las personas que han usado las plataformas colaborativas ya han

${ }^{40}$ Ver http://www.comisiondeproductividad.cl/wp-content/uploads/2018/06/Cap\%C3\%ADtulo-VI-Telemedicina.pdf.

${ }^{41}$ Ver http://www.expansion.com/economia/2016/07/30/579c78e046163f43098b4572.html.

${ }^{42}$ Los principales indicadores actualizados de la economía colaborativa en Europa están disponibles en la página «Single Market Scoreboard» de la Unión Europea. http://ec.europa.eu/internal_market/scoreboard/ performance_per_policy_area/collaborative-economy/index_en.htm.

${ }^{43}$ Ver https://data.europa.eu/euodp/data/dataset/S2112_438_ENG. 
proporcionado un servicio al menos una vez. Esto es, más del 5 por 100 de la población de la UE ya proporciona productos y servicios a través de este tipo de plataformas.

- Un 2 por 100 de la población adulta europea trabaja más de 20 horas a la semana u obtiene al menos la mitad de sus ingresos a través de plataformas de trabajo en línea, según un informe de la Comisión Europea (Pesole et al., 2018), basado en una encuesta a 32.409 trabajadores de 14 miembros de la Unión europea. El mismo informe indica que el 11,6 por 100 de los adultos españoles ha trabajado alguna vez para plataformas como Deliveroo, Glovo o Uber. Un 9,4 por 100 lo hace de forma frecuente ${ }^{44}$.

- Según el Panel de Hogares CNMC 2017 (CNMC, 2017) el 34,1 por 100 de los internautas utiliza las plataformas de economía colaborativa en España al menos una vez al mes. El informe refleja que las plataformas colaborativas más empleadas son las de compra o alquiler de productos de segunda mano, ya que casi un 27 por 100 de los internautas las usan al menos una vez al año, seguidas del alojamiento en casa de otro particular $(9,7$ por 100$)$ y de las que facilitan compartir trayectos en automóvil entre ciudades (6,4 por 100).

- En un estudio de la consultora The Cocktail Analysis (The Cocktail Analysis, 2018), con una muestra de más de 1.500 adultos en España, el 79 por 100 de los encuestados usa alguna de las plataformas digitales. El 87 por 100 conoce alguna de las plataformas, siendo Wallapop la más conocida.

- En España, BlablaCar ha crecido un 67 por 100 en los dos últimos años (20162018) y alcanza 5 millones de usuarios ${ }^{45}$.

Son cifras que no aportan un dato único y preciso del uso de estas aplicaciones, pero que sí nos indican un alto grado de aceptación, uso y satisfacción en España y la Unión Europea.

\section{Principales ventajas y retos de la economía de plataformas}

\subsection{Ventajas}

El uso de las plataformas digitales como herramienta de provisión de bienes y servicios está demostrando ser muy eficiente desde varios puntos de vista:

- Desde un punto de vista de gestión empresarial el modelo de negocio basado en coordinar recursos distribuidos (plataformas o network orchestrators) está

${ }^{44}$ Ver https://www.lavanguardia.com/economia/20180630/45490233264/trabajadores-plataformas-digitales-espana-ue-servicios-online.html.

${ }^{45}$ Ver http://www.europapress.es/motor/coches-00640/noticia-blablacar-crece-67-dos-ultimos-anos-espana-alcanza-millones-usuarios-20180618122245.html. 
demostrando ser netamente superior a los modelos basados en un capitalismo industrial tradicional de gestión de recursos centralizados y crecimiento interno. El artículo «What Airbnb, Uber and Alibaba have in common» (Libert, Wind y Fenley, 2014) destaca que los network orchestrators generan más valor y obtienen mayores beneficios y ganancias.

- Desde un punto de vista de la sociedad y el medio ambiente, la economía de plataformas tiene varias derivadas positivas. Se consigue hacer más con menos recursos haciendo un uso más eficiente de los mismos. Se promulga el principio que el acceso es mejor que la propiedad ${ }^{46} \mathrm{y}$ se crea mayor abundancia en la sociedad (absoluta cuando los bienes son digitales y relativa cuando los bienes o servicios son físicos). Los ciudadanos se convierten en agentes económicos a pequeña escala que son capaces de generar e intercambiar valor con otros ciudadanos de manera directa. En muchos casos realizarán estos intercambios por dinero (alquiler, compraventa) aunque el abanico de posibilidades incluye también el intercambio (bien sea directo o bien sea triangulado a través de alguna moneda social o sistema similar) y el regalo o préstamo gratuito. Con estos mecanismos se promulga la autosuficiencia a nivel de pequeños grupos y a la vez se refuerza la noción de interdependencia con el conjunto de la sociedad.

Finalmente hay una derivada medioambiental positiva al conseguir reducir la necesidad de fabricar más bienes y/o de construir más infraestructuras. Un ejemplo mencionado habitualmente es BlaBlaCar (ESADE, 2016) que permite optimizar el uso de los asientos vacíos en coches que están ya circulando y sin necesidad de ampliar la capacidad de las carreteras ni añadir más vehículos. Estudios del impacto de la segunda mano en el medioambiente ${ }^{47}$ también arrojan datos interesantes.

Por el lado de los críticos, existen estudios (Demailly y Novel, 2014) que ponen en tela de juicio estos impactos medioambientales hasta que se puedan cuantificar y aportar más datos concretos.

\subsection{Retos}

A la vez que presenta oportunidades, la economía de plataformas también implica retos y desafíos.

El primer reto es el propio conocimiento de estas plataformas por parte de la población en general. Este conocimiento varía mucho en las diferentes regiones. En el informe Economía colaborativa en América Latina (Fund y School, 2016), un 60 por 100 de los fundadores de startups mencionan el desconocimiento como la principal barrera para el crecimiento, muy por delante de la financiación o incluso la regulación. La situación es diferente en España para las plataformas más conocidas

\footnotetext{
46 Ver http://kk.org/thetechnium/better-than-own.

47 Ver Second Hand Effect https://secondhandeffect.schibsted.com.
} 
(Wallapop, BlaBlaCar y Uber tienen índices de notoriedad por encima del 50 por 100) (The Cocktail Analysis, 2018), pero las plataformas de nueva creación se enfrentan siempre a este reto.

Al mismo tiempo existe una barrera de inclusividad para el acceso y uso de las plataformas, al basarse, en la mayoría de casos, en herramientas tecnológicas. Sea por acceso físico a la tecnología (un teléfono móvil inteligente y conectividad) y/o bien por la capacidad de uso de la misma (por cultura digital y por la capacidad de generar confianza en desconocidos), existe una brecha digital importante para un segmento considerable de la población. En Milán han creado una Sharing Economy $S c h o o l^{48}$ con el fin de realizar una alfabetización digital en la región. En Estados Unidos se ha lanzado recientemente la iniciativa Digital Workforce Development Initiative $^{49}$ para ayudar y acompañar a las personas que trabajan mediante plataformas digitales. Las personas que no sepan usar una plataforma digital para encontrar trabajos y tareas corren el riesgo de quedar más excluidas del sistema laboral en trabajos donde antes no eran necesarias estas habilidades (Schor, 2017).

Otro reto a considerar es el abuso o el mal uso de la tecnología. El filósofo francés Paul Virilio habla del «accidente integral» (Virilio, 2007, p. 10) cuando cada tecnología, cada invento crea su(s) propio(s) accidente(s). El autor lo ejemplifica diciendo que al inventar el barco, se inventó a la vez el hundimiento. Con el uso de las plataformas digitales aún estamos comprendiendo qué tipo de accidentes o retos sociales, económicos e incluso medioambientales estamos creando. Su rápido crecimiento dificulta esta comprensión y su encaje normativo. Buenadicha, Cañigueral y De León (2017) identifican los siguientes puntos como los más críticos en el ámbito del encaje normativo:

- Comprender el buen uso y las limitaciones de la autorregulación de las plataformas. Sus mecanismos de gobernanza interna crean una buena autorregulación, pero no ocurre lo mismo con sus externalidades negativas en otros ámbitos de la sociedad y la economía.

- Coordinar varios niveles administrativos (local, regional, nacional, supranacional) en los ámbitos donde cada uno tiene competencias asignadas. Desde la promoción del mercado único digital a nivel europeo y normativas de uso de datos como la General Data Protection Regulation (GDPR) pasando por aspectos laborales o fiscales de carácter nacional, obtención de licencias de actividad de carácter regional o local, etc.

- Garantizar una competencia justa y respetar los sistemas de permisos y licencias bajo los cuales muchos sectores económicos operan hoy en día, a la vez que no se limita de manera artificial la entrada de nuevos actores en un sector productivo.

\footnotetext{
${ }^{48}$ Ver https://italy.airbnbcitizen.com/first-sharing-economy-school-milan/.

${ }^{49}$ Ver https://www.dwdi.org/.
} 
- Proteger a los consumidores en ámbitos como los derechos de consumo y resolución de conflictos, protección de datos personales, promoción de sellos de buenas prácticas, etc. En España la Organización de Consumidores y Usuarios (OCU) viene trabajando en este ámbito desde 2014. La Agencia Catalana del Consumo, dentro de la Generalitat de Catalunya, ha publicado un «Código de buenas prácticas para plataformas digitales $»^{50}$.

- Aplicar y adaptar, si es necesario, la legislación fiscal a la actividad económica que se genera a partir del uso de las plataformas digitales. Ya hace años que Hacienda trabaja en este sentido, pero a menudo el ámbito de las licencias y la falta de diálogo entre las plataformas y las administraciones dificulta los acuerdos en materia fiscal. Por otra parte algunas de las plataformas de carácter más internacional hacen uso de mecanismos de ingeniería financiera para reducir y/o eludir el pago de impuestos en el territorio donde se realiza la actividad ${ }^{51}$.

Probablemente el reto más complejo es el relativo a la normativa laboral. Esta se desarrolló para modelos de economía industrial y contratos de larga duración de 40 horas de trabajo a la semana para un único empleador. Con las plataformas digitales los ciudadanos pueden generar ingresos usando sus bienes y/o su tiempo y habilidades de manera discontinua, sin contratos, con un jefe que es un algoritmo que se ejecuta en otro país, con un nivel de subordinación distinto en cada plataforma, etc. Se necesita una profunda revisión de cómo no perder derechos laborales por el hecho de digitalizar las relaciones laborales.

Frente a esta complejidad, los reguladores deberán desarrollar estrategias para desarrollar y acompañar con una actitud proactiva la regulación de las grandes plataformas de impacto global que estarán funcionando en el territorio local, así como fomentar el diálogo y obtener datos del uso de las mismas para poder regular de manera informada. Otra estrategia recomendable es apoyar el desarrollo de plataformas de carácter más local, a menudo más conscientes de todos sus impactos económicos y sociales en el territorio. Finalmente habrá que actualizar la propia administración pública para ofrecer interfaces digitales que estas plataformas puedan usar para el pago de impuestos y/o el intercambio de datos ${ }^{52}$.

Lo imprescindible en cualquier caso es no dejar de hacerse las preguntas necesarias. No quedar paralizados por el análisis y buscar mecanismos para avanzar y aprender todos juntos como sociedad, garantizando que el progreso tecnológico deriva también en progreso social. En la siguiente sección del documento se profundiza en estos aspectos.

\footnotetext{
${ }^{50} \mathrm{Ver}$ http://economia.gencat.cat/web/.content/70_economia_catalana/arxius/economia-collaborativa/ codigo-buenas-practicas.pdf

${ }_{51}$ Ver Airbnb, Uber y BlaBlaCar tributan fuera de España por su actividad real. Ver https://www.abc. es/economia/abci-airbnb-uber-y-blablacar-tributan-fuera-espana-actividad-real-201608221106_noticia.html

52 Para el ámbito de la innovación regulatoria, el informe «Soluciones y buenas prácticas en Innovación Regulatoria« aporta una buena introducción. Ver https://www.slideshare.net/acanyi/soluciones-y-buenas-prcticas-en-innovacin-regulatoria
} 


\section{Hacia una economía de plataformas socialmente responsable}

\subsection{Amplia diversidad de actores y propósitos en la economía de plataformas}

La economía de plataformas se fundamenta en crear espacios de encuentro (digitales en su mayoría) donde las personas con necesidades/intereses comunes realizan intercambios de valor (mediante plataformas que permite comprar, vender, alquilar, prestar, intercambiar, hacer trueque o regalar) y/o colaboran para un objetivo común (comunidades digitales). El uso de tecnologías de la información aporta la confianza y reciprocidad necesaria, a la vez que se reducen de manera significativa los costes de coordinación y transacción.

Los medios de comunicación generalistas, así como muchos de los informes acerca del fenómeno de la economía de plataformas, tienden a simplificar de manera excesiva los actores de la economía de plataformas, focalizándose únicamente en las startups de carácter más comercial.

Las plataformas son, en el fondo, herramientas digitales que permiten codificar (mediante los algoritmos y las funciones disponibles en las aplicaciones) acuerdos entre partes de un ecosistema ${ }^{53}$ de manera altamente escalable. En un mundo de alta conectividad, esta necesidad de coordinación ecosistémica es aplicable a cualquier ámbito social y económico, con lo que la economía de plataformas también puede ser de utilidad a sectores más sociales (cooperativas, organizaciones sin ánimo de lucro, economía social y solidaria, Empresas $\mathrm{B}^{54}$, etc.) así como al propio sector público. En la economía de plataformas el sector público puede ejercer un rol de agente productor (ejemplo: poniendo a disposición, por la tarde, coches municipales en un plataforma de alquiler de coches o un sistema de carsharing ${ }^{55}$ ), agente consumidor (ejemplo: realizando campañas de matchfunding ${ }^{56}$ para apoyar a proyectos ciudadanos) o incluso un rol de promotor y proveedor de la plataforma digital (ejemplo: alquilando espacios municipales mediante una plataforma propia al estilo Airbnb ${ }^{57}$ ).

La economía de plataformas tiene una gran diversidad de actores que resumimos, en función de su propósito organizacional, en el Cuadro 1.

Esta discriminación en base al propósito/rol de los diversos actores de la economía de plataformas es relevante para un análisis que permita crear políticas públicas diferenciadas para incentivar la tipología de actividades que las administraciones consideren más oportunas (economía de plataformas «responsable») y desincentiven o dificulten aquellas que se consideren menos adecuadas para el bien común.

\footnotetext{
53 Ver The Emerging Art of Ecosystem Management. Ver https://www.bcg.com/publications/2019/emerging-art-ecosystem-management.aspx.

54 Ver https://sistemab.org/.

55 Ver punto 4.3 en Momo Car-Sharing - Guideline for municipalities and governments. Ver http://bit. ly/2MJWurv.

56 Ver https://www.goteo.org/matchfunding.

57 Ver https://www.shareable.net/blog/amsterdam-to-launch-airbnb-style-rentals-of-municipal-buildings.
} 


\section{CUADRO 1}

ACTORES DE LA ECONOMÍA COLABORATIVA

\begin{tabular}{|c|c|c|}
\hline Propósito/Rol & Definición & Ejemplos \\
\hline Con ánimo de lucro & $\begin{array}{l}\text { Entidades con ánimo de lucro que rea- } \\
\text { lizan actividades de compra/venta, al- } \\
\text { quiler/préstamo, intercambio, trueque } \\
\text { o regalo/donación con la ayuda de tec- } \\
\text { nologías de la información que reducen } \\
\text { de manera considerable los costes de } \\
\text { transacción y facilitan los intercambios } \\
\text { entre desconocidos }\end{array}$ & $\begin{array}{l}\text { Internacionales: } \\
\text { - Airbnb } \\
\text { - BlaBlaCar } \\
\text { - Ebay } \\
\text { - Car2Go } \\
\text { - Zipcar } \\
\text { Locales: } \\
\text { - Wallapop } \\
\text { - SocialCar } \\
\text { - Percentil } \\
\text { - Verkami }\end{array}$ \\
\hline $\begin{array}{l}\text { Empresa social/ } \\
\text { Cooperativa/ } \\
\text { Empresas B }\end{array}$ & $\begin{array}{l}\text { La misma definición, excepto que las } \\
\text { motivaciones sociales y medioambien- } \\
\text { tales tienen prioridad respecto al lucro } \\
\text { económico. En las cooperativas, la } \\
\text { propiedad es compartida. }\end{array}$ & $\begin{array}{l}\text { - SomMobilitat (coop) } \\
\text { - La Colmena que dice Sí } \\
\quad \text { (gobernanza inclusiva) } \\
\text { - Ecrowd (Empresa B) } \\
\text { - Ulule (Empresa B) }\end{array}$ \\
\hline Sin ánimo de lucro & $\begin{array}{l}\text { La misma definición que la empresas } \\
\text { con ánimo de lucro, excepto que son } \\
\text { actores sin ánimo de lucro donde la } \\
\text { motivación principal es avanzar en su } \\
\text { misión y/o propósito }\end{array}$ & $\begin{array}{l}\text { - Goteo (Fundación) } \\
\text { - Freecycle } \\
\text { - Reutiliza.cat } \\
\text { - NoLoTiro.org } \\
\text { - YoNoDesperdicio }\end{array}$ \\
\hline Comunidad & $\begin{array}{l}\text { Actores focalizados en la escala local } \\
\text { y/o de barrio, con una diversidad de es- } \\
\text { tructuras legales, aunque las entidades } \\
\text { sin ánimo de lucro y modelos informa- } \\
\text { les son los más habituales. La mayoría } \\
\text { de transacciones no se monetizan. } \\
\text { El uso de las tecnologías de la informa- } \\
\text { ción es más modesto con un énfasis en } \\
\text { el contacto y las relaciones humanas. } \\
\text { A menudo de manera explícita tienen } \\
\text { un foco en conseguir la sostenibilidad } \\
\text { en el ámbito local. }\end{array}$ & $\begin{array}{l}\text { - Jardines comunitarios } \\
\text { - Grupos de consumo de proximidad } \\
\text { - Bancos de tiempo } \\
\text { - EcoXarxes } \\
\text { - El Campo de la Cebada en Madrid } \\
\text { - PetitBus }\end{array}$ \\
\hline Sector público & $\begin{array}{l}\text { A diferencia de los casos anteriores, las } \\
\text { entidades del sector público están suje- } \\
\text { tas a rigurosos estándares de responsa- } \\
\text { bilidad, transparencia y legitimidad. } \\
\text { Pueden usar su infraestructura más } \\
\text { sofisticada para apoyar o crear acuer- } \\
\text { dos con el resto de actores, para pro- } \\
\text { mocionar nuevas formas de compartir } \\
\text { la ciudad, aunque tienen que responder } \\
\text { a los intereses de todos los ciudadanos } \\
\text { (algunos de los cuales no estarán invo- } \\
\text { lucrados en estas actividades) y a órga- } \\
\text { nos de gobierno superiores. }\end{array}$ & $\begin{array}{l}\text { - } \text { Bibliotecas municipales } \\
\text { - Servicios de bicicleta compartida } \\
\text { pública } \\
\text { - Reparat millor que nou (AMB) } \\
\text { - Renova la teva roba (Ayuntamiento } \\
\text { de Barcelona) } \\
\text { - Compra pública con criterios en fa- } \\
\text { vor de los actores de la ciudad co- } \\
\text { laborativa } \\
\text { - Monedas locales con apoyo muni- } \\
\text { cipal }\end{array}$ \\
\hline
\end{tabular}

FUENTE: Adaptación de Local Governments Sharing Economy. Véase http://www.localgovsharingecon.com/. 


\subsection{De la crítica a la construcción con propuestas concretas}

«El mantra que ha inspirado las iniciativas digitales ha sido la agilidad y la disrupción, y eso es válido para las etapas de descubrimiento e ingeniería, pero ahora haremos bien en prestar más atención a los cambios que proponemos, porque ya estamos en etapa de transformación y eso implica que nuestras propuestas ya afectan a nuestro orden social, y por tanto a nuestros valores y a nuestra ética», nos alerta el consultor Genís Roca en un artículo ${ }^{58}$ escrito durante la huelga de taxis en España en el verano de 2018.

Hay que observar las plataformas digitales como sistemas de gobernanza. Las plataformas digitales median, y en el fondo dictan, relaciones económicas y sociales entre los participantes. Estas relaciones económicas y sociales pueden orientarse hacia un capitalismo responsable, un capitalismo extremo o hacia mecanismos del bien común. Estos aspectos entran dentro del diseño y el propósito de la plataforma.

Estamos en un momento crítico de toma de decisiones para conseguir la mejor versión posible de la economía de plataformas. Además de la crítica (necesaria y justificada) a las muchas de las propuestas de plataformas actuales ${ }^{59}$ que no alteran de manera significativa los fundamentos del capitalismo industrial, debemos explorar y proponer alternativas viables que nos permitan orientar el desarrollo de la economía de plataformas hacia el bien común y la responsabilidad social en sus actividades.

- Las plataformas digitales extractivas son aquellas que se diseñan pensando casi exclusivamente en la generación de lucro para los propietarios (shareholders) de la plataforma, quienes habitualmente son el grupo fundador y socios inversores.

- Las plataformas digitales responsables ${ }^{60}$ son aquellas que integran en el diseño de sus actividades los intereses de todos sus participantes o stakeholders, incluidos aquellos impactados por las externalidades negativas.

- La economía de plataformas procomún se refiere a un modelo emergente basado en la colaboración de comunidades de personas contribuidoras que a partir de plataformas digitales desarrollan recursos comunes cuya propiedad es compartida y deviene accesible como bien público.

En lo que queda de sección presentamos algunas de las propuestas que buscan ayudar en el diseño de plataformas digitales más socialmente responsables. La intención no es realizar un juicio de valor sobre los modelos descritos (que sería

${ }^{58} \mathrm{Ver}$ https://www.lavanguardia.com/local/barcelona/20180730/451165907765/sobre-disrupcion-digital-leyes-y-taxis.html.

${ }_{59}$ Ver http://www.shareable.net/blog/how-platform-coops-can-beat-death-stars-like-uber-to-create-a-realsharing-economy.

${ }^{60}$ Hacia una economía colaborativa responsable. Ver http://oikonomics.uoc.edu/divulgacio/oikonomics/ es/numero06/dossier/acanigueral.html 
prematuro), ni una comparación entre ellos (que no es posible), sino simplemente exponer los modelos.

\section{Sharing Business Model Compass}

Una vez reconocida la gran diversidad de negocios colaborativos existentes es importante poder ubicarlos de manera sistematizada en algún punto del espectro que hay entre las plataformas de carácter más capitalista y las plataformas de carácter más cooperativista (este aspecto se desarrolla más adelante).

Es necesario también dar guías a los emprendedores interesados en empujar el desarrollo económico y social a nivel local o regional mediante el uso eficiente y responsable de las plataformas digitales como herramientas.

El profesor Boyd Cohen y Pablo Muñoz han sido capaces de identificar un buen número de dimensiones que están frecuentemente consideradas como aspectos clave de las startups de economía de plataformas y han creado el modelo Sharing Business Model Compass, que permite organizar las iniciativas de manera coherente y sistematizada.

No es una herramienta de clasificación sencilla, sino un modelo dinámico para mapear los esfuerzos de las empresas que aspiran a entrar en el espacio de la economía de plataformas.

El Sharing Business Model Compass (Figura 2) consta de seis dimensiones distintas, que son únicas para los modelos de negocio de economía de plataformas: Tecnología, Transacción, Enfoque de Negocio, Recursos Compartidos, Modelo de Gobernanza y Tipo de Plataforma. Cada dimensión tiene tres grados, representando una forma continua desde, por ejemplo, los modelos de gobierno con propiedad y control concentrados, hasta una propiedad y control completamente distribuidos.

\section{Cooperativismo de plataforma}

¿Qué pasaría si las plataformas compartieran propiedad e incluso beneficios con los usuarios que, de hecho, son los encargados de generar valor dentro de ellas ?61

El término Platform Coop ${ }^{62}$ (Scholz y Schneider, 2014) o cooperativismo de plataforma (como se traduce al español) propone una economía de plataformas que combina lo mejor de las plataformas digitales con los principios cooperativistas internacionales. Esto es, con la idea de propiedad compartida, la puesta en marcha de nuevos modelos de gobernanza, la participación del ciudadano productor de valor en la toma de decisiones en principios de igualdad (frente al control exclusivo del inver-

\footnotetext{
${ }^{61}$ Ver https://www.fastcompany.com/3038476/collaborative-economy-companies-need-to-start-sharingmore-value-with-the-people-who-make-th.

${ }^{62}$ Ver http://platformcoop.net.
} 


\section{FIGURA 2}

\section{SHARING BUSINESS MODEL COMPASS}

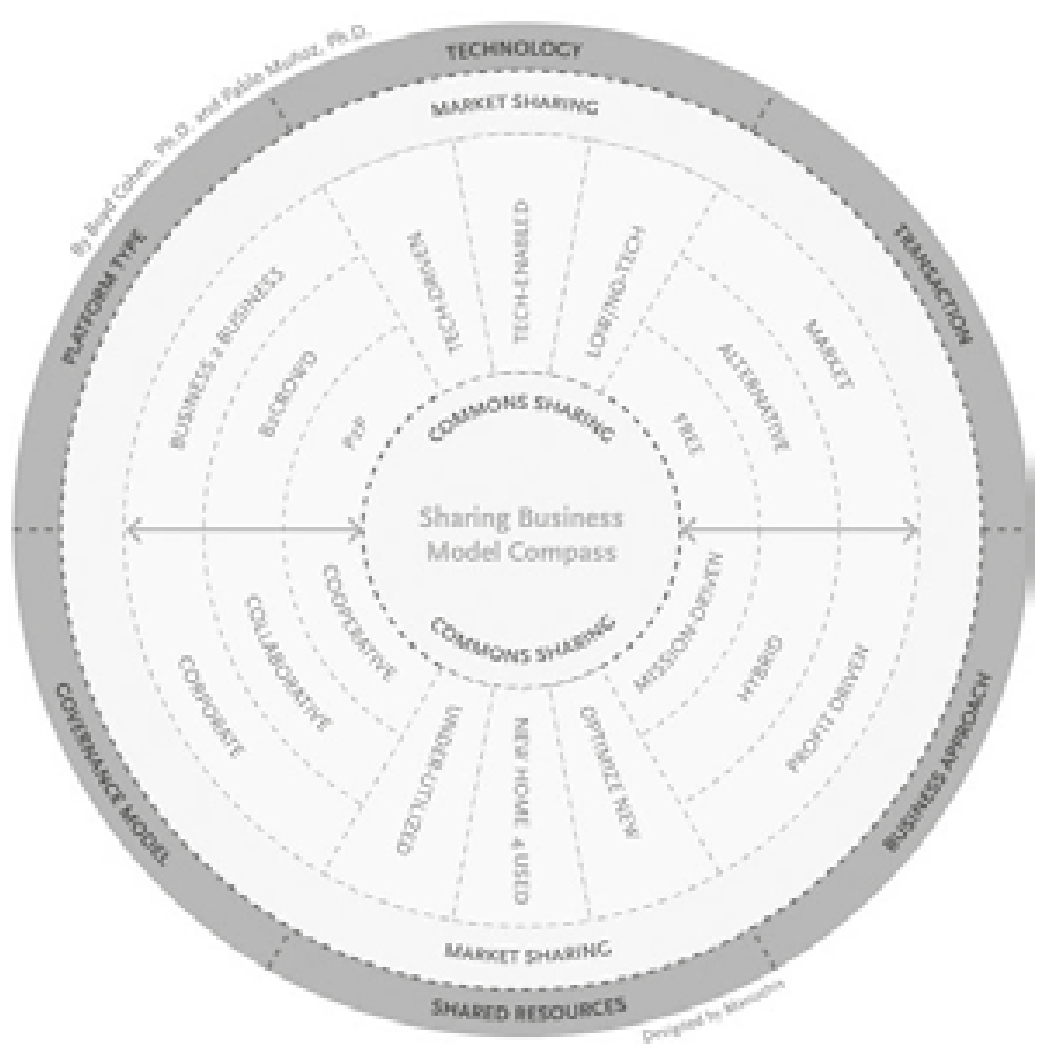

FUENTE: Cohen y Muñoz (2016). The sharing business model compass.

sor en las plataformas capitalistas) y con el reparto de los beneficios entre los usuarios.

Scholz (2016) concluye que la economía de plataformas corporativa «no es simplemente una continuación del capitalismo predigital tal como lo conocemos, hay notables discontinuidades, un nuevo nivel de explotación y una mayor concentración de la riqueza». Y añade que el cooperativismo de plataforma permite dejar de depender «de las infraestructuras digitales que están diseñadas para extraer provecho para un número muy reducido de propietarios de plataformas y accionistas».

Scholz apunta a diez principios para el cooperativismo de plataforma: la propiedad colectiva de la plataforma; el pago decente y la seguridad de renta; la transparencia y portabilidad de los datos; la apreciación y el reconocimiento del valor generado; las decisiones colectivas en el trabajo; un marco legal protector; la protección transferible de los trabajadores y la cobertura de las prestaciones sociales; la protec- 
ción frente a las conductas arbitrarias en los sistema de rating; el rechazo a la excesiva vigilancia en el lugar de trabajo; y, por último, el derecho de los trabajadores a desconectar.

Ya existen ejemplos de plataformas de éxito en esta dirección. Fairmondo: es una cooperativa alemana con más de 2.000 socios que se ha diseñado para convertirse en la alternativa de eBay -los vendedores son copropietarios de la organización. Los fotógrafos de Stocksy han constituido el equivalente a una cooperativa de trabajo que les permite decidir cómo se gestiona la empresa y obtener más beneficios por foto vendida a través de internet. Desde España, SomMobilitat (cooperativa que promueve la movilidad eléctrica y colaborativa) es referente internacional.

Marco para evaluar las cualidades democráticas en plataformas de economía colaborativa

Desde el grupo de trabajo BarCola (Barcelona Colaborativa) y Dimmons (Universitat Oberta de Catalunya) se ha venido trabajando desde 2015 en la definición y el desarrollo de un marco de referencia para evaluar las plataformas digitales. Fuster Morell y Espelt (2018) muestran el modelo más avanzado y detallado hasta el momento. La síntesis de este modelo se presenta en el gráfico acerca de cualidades procomún para plataformas colaborativas (Figura 3 ).

\section{FIGURA 3}

\section{ESTRELLA DE CUALIDADES PROCOMÚN PARA PLATAFORMAS COLABORATIVAS}

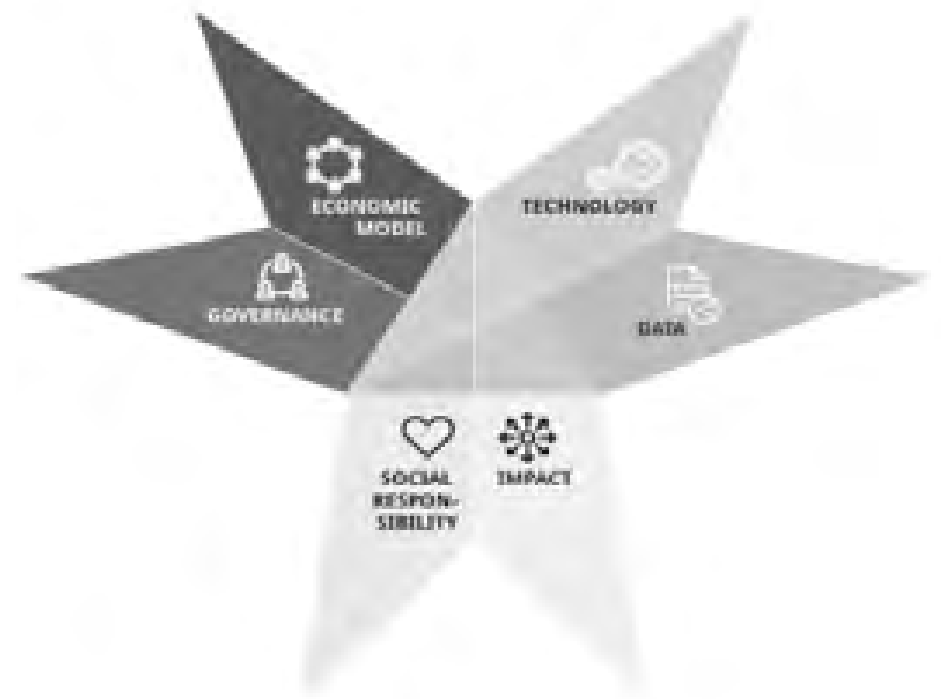

FUENTE: Morell y Espelt (2018). 
Las cualidades democráticas de la economía colaborativa se articulan alrededor de tres dimensiones principales, con seis subdivisiones:

- Gobernanza y modelo económico: estas dos subdimensiones están interconectadas. Por tanto, la forma en que se rige el proyecto o la plataforma está conectada con el modelo económico subyacente que esta promueve.

- Políticas de conocimiento y tecnología: en el mismo sentido, las políticas de conocimiento y tecnología están interconectadas. Por tanto, la adopción de ciertas herramientas o licencias tecnológicas afectará a la forma en que la plataforma promueve el conocimiento.

- Responsabilidad social e impacto. Estas dimensiones se relacionan con las externalidades y los impactos negativos, como la exclusión social y las desigualdades sociales o la inclusión de género en el acceso a la plataforma. Este aspecto se refiere igualmente al cumplimiento de estándares de salud y seguridad que protegen al público y al medioambiente. Este aspecto también se refiere a la protección del interés general, el espacio público y los derechos humanos básicos, como el acceso a la vivienda.

\section{Diseñando plataformas positivas}

El estudio «Diseñando plataformas positivas desde un modelo de gobernanza inclusivo ${ }^{63}$ define cinco principios de diseño para desarrollar modelos de gobernanza positivos en las plataformas digitales. Estos principios pretenden ser una guía para crear plataformas responsables e inclusivas.

- Inclusión: Inclusión del máximo número de stakeholders en la toma de decisiones, asegurando que los contribuidores más activos de la plataforma, tengan igual o más poder para tomar decisiones estratégicas que el resto de stakeholders.

- Participación: Incorporación de los valores de justicia, sencillez, transparencia y confianza en todos los procesos de toma de decisión de la plataforma para fomentar la participación.

- Autonomía: Introducción de la autonomía en la toma de decisiones para mantener la agilidad mientras se escala, pero asegurando que todos los contribuidores afectados por esas decisiones puedan participar de alguna manera.

- Reconocimiento del valor generado: Desarrollo de un sistema que permita entender qué es valor en el contexto de la plataforma, y basándose en ello, proporcionar una serie de beneficios alineados con las expectativas e intereses de los profesionales.

\footnotetext{
${ }^{63}$ Ver https://www.ouishare.net/article/disenando-plataformas-positivas.
} 
- Bienestar: Inclusión de un sistema de protección y seguridad en el modelo de gobernanza de la plataforma acorde con el concepto de bienestar entendido por los profesionales que participan en ella.

En esta sección cuarta hemos revisado una serie de marcos teóricos que nos permiten observar y analizar la realidad de las plataformas digitales en toda su complejidad de actores, propósitos y decisiones de diseño en relación a modelos económicos, modelos de gobernanza e incluso políticas de uso de la tecnología subyacente, datos que circulan por las plataformas y el ecosistema que se genera a su alrededor.

\section{Conclusiones}

La economía de plataformas nos dibuja un futuro, que de hecho es más presente que futuro, que ofrece una manera más eficiente de organizar los recursos con potenciales beneficios en el ámbito de lo económico, lo social y lo medioambiental. A su vez, la velocidad y la escala del fenómeno hacen difícil afrontar los numerosos retos y accidentes que estos nuevos modelos de organización generan.

Para poder ir más allá de hacer simplemente el capitalismo más eficiente-«capitalismo de plataforma» ha sido el término popularizado por el autor Nick Srnicek (Srnicek, 2016)-, habrá que:

- Reconocer la complejidad del ecosistema de actores de la economía de plataformas y sus diversos propósitos (startups con ánimo de lucro, cooperativas/ empresas sociales, organizaciones sin ánimo de lucro, las propias administraciones, etc.) y acompañar el desarrollo de políticas públicas.

- Comprender que estamos en un intervalo de reacomodo de la revolución (Pérez, 2003) que representa la economía de plataformas. En estos períodos se deben tomar decisiones socio-políticas para orientar el mejor uso de la tecnología en pro del beneficio común. Es el momento de ser valientes y exigentes porque son decisiones que van a definir en gran medida los contratos sociales de las próximas décadas.

- Trabajar sobre modelos y propuestas concretas que nos permitan añadir el adjetivo «responsable» a las palabras «Economía de plataformas». En este artículo se han presentado algunos de estos modelos como inspiración.

Nadie tiene las respuestas a muchas de las preguntas que nos plantean las plataformas digitales. En cualquier caso todos tenemos el deber compartido de buscar las mejores respuestas en pro del bien común.

Este artículo quiere ser un pequeño grano de arena a esta labor de exploración colectiva. 


\section{Referencias bibliográficas}

Buenadicha, C., Cañigueral, A., \& De León, I. L. (2017). Retos y posibilidades de la economía colaborativa en América Latina y el Caribe. Inter-American Development Bank. https://doi.org/10.18235/0000707.

CNMC (2017, 2 de noviembre). Panel de hogares CNMC: enganchados a las "apps" de compraventa de segunda mano-CNMC blog. Recuperado de https://blog.cnmc. es/2017/11/02/panel-de-hogares-cnmc-enganchados-las-apps-de-compraventa-de-segunda-mano/.

Demailly, D., \& Novel, A.-S. (2014). The sharing economy: make it sustainable. Studies (3), 14-30.

ESADE (2016, 29 de junio). Antena de Innovación Social. Nosotros compartimos. ¿Quién gana?: Controversias sobre la economía colaborativa. Recuperado de https://www.slideshare.net/ESADE/antena-de-innovacion-social-controversias-economia-colaborativa.

Fuster, M., \& Espelt, R. (2018). A Framework for Assessing Democratic Qualities in Collaborative Economy Platforms: Analysis of 10 Cases in Barcelona. Urban Science, 2(3), 61 .

Libert, B., Wind, Y., \& Fenley, M. (2014). What Airbnb, Uber, and Alibaba have in common. Harvard Business Review. Recuperado de https://www.openmatters.com/s/ WhatAirbnbUberandAlibabaHaveinCommon.pdf.

Multilateral Investment Fund \& I. E. Business School (2016). Economía colaborativa en América Latina. Inter-American Development Bank. Recuperado de https://publications. iadb.org/handle/11319/7806.

Pérez, C. (2003). Technological Revolutions and Financial Capital: The Dynamics of Bubbles and Golden Ages. Northampton, EE. UU.: Edward Elgar Publishing.

Pesole, A., Urzi-Brancati, M. C., Fernández-Macías, E., Biagi, F., \& González-Vázquez, I. (2018). Platform Workers in Europe Evidence from the COLLEEM Survey. Publications Office of the European Union.

Rodríguez, S. (2017). Los modelos colaborativos y bajo demanda en plataformas digitales. Sharing España y Asociación Española de La Economía Digital.

Scholz, T. (2016). Cooperativismo de plataforma. São Paulo, Brasil: Elefante. Recuperado de https://endefensadelsl.org/cooperativismo_de_plataforma-binder.pdf.

Schor, J. B. (2017). Does the sharing economy increase inequality within the eighty percent?: findings from a qualitative study of platform providers. Cambridge Journal of Regions, Economy and Society, 10(2), 263-279.

Srnicek, N. (2016). Platform Capitalism. Wiley.

The Cocktail Analysis (2018, 3 de abril). Economía de plataformas. Recuperado de https:// www.slideshare.net/TCAnalysis/economia-de-plataformas.

Virilio, P. (2007). The Original Accident. Polity. 